\title{
The minimum clinically significant difference in visual analogue scale pain score does not differ with severity of pain
}

\author{
A-M Kelly
}

\begin{abstract}
Objective-To determine whether minimum clinically significant difference in visual analogue scale (VAS) pain score varies according to the severity of pain reported.

Method-Prospective descriptive study of adult patients in an urban emergency department (ED). On presentation to the ED, patients marked the level of their pain on a $100 \mathrm{~mm}$, non-hatched VAS scale. At 20 minute intervals thereafter they were asked to give a verbal categorical rating of their pain as "a lot better", "a little better", "much the same", "a little worse" or "much worse" and to mark the level of pain on a VAS scale of the same type as used previously. It was pre-defined that patients with VAS pain scores of $\mathbf{3 0}$ $\mathrm{mm}$ or less would be categorised as having mild pain, those with scores of $70 \mathrm{~mm}$ or more were categorised as having severe pain and those from $31 \mathrm{~mm}$ to $69 \mathrm{~mm}$, moderate pain. The minimal clinically significant difference (MCSD) in VAS pain score was defined as the mean difference between current and preceding scores when the subject reported "a little worse" or "a little better" pain.
\end{abstract}

Results-156 patients were enrolled in the study, yielding 88 evaluable comparisons where pain was rated as "a little better" or "a little worse". The MCSD in VAS score in the group overall was $12 \mathrm{~mm}(95 \% \mathrm{CI}$ $9 \mathrm{~mm}$ to $15 \mathrm{~mm}$ ). MCSD in VAS score for the "mild pain" group was $11 \mathrm{~mm}(95 \% \mathrm{CI}$ $4 \mathrm{~mm}$ to $18 \mathrm{~mm}$ ), for the "moderate pain" group $14 \mathrm{~mm}(95 \% \mathrm{CI} 10 \mathrm{~mm}$ to $18 \mathrm{~mm})$ and for the severe pain group, $10 \mathrm{~mm}$ $(95 \%$ CI $6 \mathrm{~mm}$ to $14 \mathrm{~mm})$. There is no statistical difference between the MCSD in VAS score between the severity groups. Conclusions-The MCSD in VAS pain score does not differ with the severity of pain being experienced.

(Emerg Med f 2001;18:205-207)

Emergency Medicine, Western Hospital, Melbourne, Australia

Correspondence to:

Professor Kelly, Department of Emergency Medicine, Western Hospital, Private Bag, Footscray, Vic 3011, Australia

(Anne-Maree.Kelly@)

nwhen.org.au)

Accepted for publication 4 September 2000

health care workers in emergency departments (ED). However, to assess the quality of current pain management efforts and to evaluate new pain management techniques, pain must be measured, the results analysed and differences assessed for both statistical and clinical significance.
The methodology most commonly used for the evaluation of pain severity and relief, is the visual analogue scale (VAS). ${ }^{1}$ It is easy to use, provides reproducible results and is applicable to a variety of practice settings. ${ }^{2}$ It is also sensitive to treatment effects and the data derived can be analysed using parametric statistical techniques. $^{3{ }^{4}}$ The main disadvantage of this methodology is that there is limited information to correlate these statistically significant results with clinical significance to patients in terms of reduction of their pain experience. With respect to emergency medicine pain research, the challenges are to determine the minimal clinically significant difference (MCSD) in pain experience (as measured by VAS pain scores) and to determine if this measure differs according to variables such as age, sex, cause of pain and severity of pain.

Two previous studies have attempted to ansswer this question for acute pain in the ED setting. These found the MCSD in VAS pain scores to be $13 \mathrm{~mm}$ and $9 \mathrm{~mm}$ respectively. ${ }^{2}{ }^{5}$ Furthermore, Kelly ${ }^{5}$ reported that the MCSD in VAS pain score did not differ with sex, age or cause of pain.

An unanswered question is whether the MCSD in VAS pain score differs with the amount of pain being experienced. This question is of importance in the interpretation of research results. If it is different for those reporting mild or moderate pain compared with those reporting severe pain, the results of studies may be influenced by the relative number of patients in each pain severity category. Also a result might be interpreted incorrectly as significant or insignificant based on an invalidly derived MCSD figure.

The aim of this study was to determine whether the MCSD in VAS pain score for acute pain in the ED setting varies with the severity of pain being experienced.

\section{Method}

SETTING

This prospective, descriptive study was conducted in the Department of Emergency Medicine at Western Hospital in Melbourne, a 500 bed community teaching hospital. The project was approved by the Research and Ethics Committee of the North Western Healthcare Network.

\section{POPULATION}

For the week beginning 1 December 1997, all patients presenting with acute pain between 8 am and midnight were eligible for entry into 
the study. Exclusion criteria were age under 16 years, inability to understand the questions in English, inability to give informed consent, inability to mark a VAS and altered level of consciousness, including intoxication.

\section{MEASUREMENTS}

On presentation to the ED and after having given informed consent, patients were asked to mark the level of their pain on a $100 \mathrm{~mm}$, nonhatched VAS scale marked at one end as "no pain" and at the other as "worst pain imaginable". At 20 minute intervals thereafter they were asked to give a verbal categorical rating of their pain as "a lot better", "a little better", "much the same", "a little worse" or "much worse" and to mark the level of pain on a VAS scale of the same type as used previously. Subjects were not permitted to refer to previous VAS markings. A maximum of three comparisons was recorded for each subject. Subjects were withdrawn from the study if they became pain free, on discharge or transfer from the ED or at their request.

Pain interventions, for example splintage, ice and pharmacological therapy, were instituted according to clinical need as assessed by the treating doctor. Pain management was not delayed or withheld by participation in this study. Interviews and data collection were performed by researchers who were independent of the clinical management of the subjects.

Before analysis of the data, pain severity categories were defined. Patients with VAS pain scores of $30 \mathrm{~mm}$ or less were defined as having mild pain. Those with scores of $70 \mathrm{~mm}$ or more, were considered to have severe pain and those from $31 \mathrm{~mm}$ to $69 \mathrm{~mm}$ moderate pain. This categorisation is based, in part, on the findings of Collins et al. ${ }^{6}$

The minimal clinically significant difference in VAS pain score was defined as the mean difference between current and preceding scores when the subject reported "a little worse" or "a little better" pain.

Sample size was difficult to estimate as there was no prior data for this group of patients to indicate the proportion of patients that would lie in each severity group (that is, the distribution of VAS pain scores) or the proportion of comparisons that would be rated as "a little better" or "a little worse". Based on Todd's study, ${ }^{2}$ the proportion of patients giving these comparisons would be about $30 \%$. That work however did not report the standard deviation of the scores. The sample size of 156 represents a compromise between what was practical in the time frame available and the expectation of 450 evaluable comparisons ( 2.5 per patient) of which about 150 would be "a little more" or "a little less".

\section{Results}

A total of 156 adult subjects were enrolled in the study. Twenty two were withdrawn from analysis because of inadequate documentation, protocol violation or patient request.

There were 88 evaluable comparisons where pain, compared with the previous measure, was categorised a "a little better" or "a little
Table 1 Minimum clinically significant difference in VAS pain score for each pain severity group

\begin{tabular}{llll}
\hline Severity group & $\begin{array}{l}\text { Number of } \\
\text { subjects }\end{array}$ & $\begin{array}{l}\text { MCSD in VAS } \\
\text { score }(\mathrm{mm})\end{array}$ & $\begin{array}{l}\text { 95\% CI } \\
(\mathrm{mm})\end{array}$ \\
\hline Mild & 26 & 11 & 4 to 18 \\
Moderate & 39 & 14 & 10 to 18 \\
Severe & 23 & 10 & 6 to 14 \\
\hline
\end{tabular}

worse". If it is assumed that the degrees of pain comparison categorised as "a little worse" or "a little better" are equivalent, the MCSD in VAS pain score in this group is $12 \mathrm{~mm}(95 \% \mathrm{C} 1$ $9 \mathrm{~mm}$ to $15 \mathrm{~mm}$ ). To combine the comparisons, the positive or negative sign for pain score differences when the subject reported that pain had decreased was reversed.

The minimum clinically significant difference in VAS pain score for each pain severity category calculated using the same methodology as described above is shown in table 1 . There is no statistical difference between the MCSD in VAS score between the severity groups.

\section{Discussion}

To improve the quality of pain management and to evaluate new pain management techniques, pain must be measured, the results analysed and changes assessed for clinical significance. It is this latter task, the assessment of clinical significance, which poses the biggest challenge.

Because of its practicality, reproducibility, sensitivity to treatment effects and ease of analysis, the VAS pain scale is a powerful research tool in the field of pain research. ${ }^{1}$ Despite this, it could be tempting to overestimate the clinical importance of small differences in scores because they reach statistical significance. Clinical significance and statistical significance are not necessarily the same and it is the clinical impact on our patients and our practice that is the more important.

Two previous studies have attempted to define the degree of change on a VAS pain scale that is clinically significant to patients. Todd et $a l^{2}$ in a study of patients with acute traumatic pain and using similar methodology to that used in this study, found the MCSD in VAS pain scale to be $13 \mathrm{~mm}(95 \% \mathrm{C} 110 \mathrm{~mm}$ to $17 \mathrm{~mm}$ ). They concluded that studies of pain experience that report less than a $13 \mathrm{~mm}$ change in pain severity (on a $100 \mathrm{~mm}$ scale), although statistically significant, may have no clinical importance. A similar study of patients with both traumatic and non-traumatic pain found the MCSD in VAS pain score to be $9 \mathrm{~mm}(95 \%$ CI $6 \mathrm{~mm}$ to $13 \mathrm{~mm}) .^{5}$ This study also analysed their data by age, sex and cause of pain and found no significant difference in MCSD in VAS pain score for each of these variables. It might be suggested that the MCSD in VAS pain score is not the same across the whole range of the scale. Results of this study suggest that this is not the case.

The impact of these findings is significant. If the MCSD in VAS pain score was different for those reporting mild or moderate pain compared with those reporting severe pain, the 
results of studies might be influenced by the relative number of patients in each pain severity category that participate in the study. Also, a result might be interpreted incorrectly as significant or insignificant based on an invalidly derived MCSD figure. The finding of this study that the MCSD in VAS score does not differ between pain severity groups will make design and interpretation of pain research easier.

This study has some limitations that must be considered in interpreting the results. The assumption that the degrees of pain comparison categorised as "a little worse" or "a little better" are equivalent may not be valid. Patients may have perceived more "room" on the scale to decrease rather than increase their pain score or vice versa. In addition, given the wide confidence intervals found, the sample size of 88 is small making a type II error possible. Unfortunately this could not be predicted at the start of the study, but will inform future researchers in sample size estimations. The sample of patients is limited to those able to communicate in English who presented to a community teaching hospital ED, thus the generalisability to other cultural groups may be questioned. In addition, patients were not given access to their previous pain ratings. Although this is common practice, it has been shown that patients may overestimate their pain if previous ratings are not available. ${ }^{7}$ There may also be an effect attributable to interpersonal relationships between the interviewer and the patient, particularly if there is a change of staff. ${ }^{8}$ In this study a small number of interviewers were used, they were independent of patient care, the method of interview was standardised and the number of patients "handed over" between researchers was very small, so this effect is unlikely to have had an impact on the findings.

In conclusion, this study found the MCSD in VAS pain score (on a $100 \mathrm{~mm}$ VAS scale) did not differ with the severity of pain being experienced.

Data collection for this study was performed by research assistants Andrew Braun, Nancy Suh, Alex Tan and Marylyn Varga. Associate Professor Steve Farish, University of Melbourne, assisted with data analysis.

Contributor

Anne-Maree Kelly initiated the study, formulated the hypothesis tested, designed the study methodology, oversaw data collection by the research assistants, analysed and interpreted the data and wrote the paper.

1 Wallerstein SL. Scaling clinical pain and pain relief. In: Bromm B, ed. Pain measurement in man: neurophysiological correlates of pain. New York: Elsevier, 1984.

2 Todd KH. Clinical versus statistical significance in the assessment of pain relied. Ann Emerg Med 1996;27:439-41.

3 Philip BK. Parametric statistics for evaluation of the visual analog scale. [Letter]. Anesth Analg 1990;71:710.

4 Dexter F, Chestnut DH. Analysis of the statistical tests to compare visual analog scale measurements among groups. Anesthesiology 1995;82:896-902.

5 Kelly AM. Does the clinically significant difference in VAS pain score differ with age, gender or cause of pain? Acad pain score differ with age, g
Emerg Med 1998;5:1086-90.

6 Collins SL, Moore RA, McQuay HJ. The visual analogue pain intensity scale: What is moderate pain in millimetres? Pain 1997;72:95-7.

7 Scott J, Huskisson EC. Graphic representation of pain. Pain 1976;2:175-84.

8 Menegazzi J. Measuring pain at baseline and over time. Ann Emerg Med 1996;27:433-5. 